\title{
EXCHANGE MODELS FOR THE EMERGENCE OF INCOME DISTRIBUTION AND ECONOMIC INEQUALITY
}

\author{
M.L. BERTOTTI \& G. MODANESE \\ Faculty of Science and Technology, Free University of Bozen-Bolzano, Piazza Universitá 5, 39100 Bolzano, Italy
}

\begin{abstract}
Also motivated by the topical problem of growing economic inequality, we propose and investigate models describing the formation process of income distribution in a closed society. Our approach fits in with a complex system perspective. We look at society as a system composed by a large number of individuals, divided into income classes. The information we derive on observable "macroscopic" features results from the interplay of a whole of interactions - money exchanges, payment of taxes, redistribution of benefits, payment of fines - occurring between individuals, i.e. at the "microscopic" level. In order to show the flexibility and the exploratory aspect of the models, we consider and compare cases characterized by different tax rates or in which means-tested welfare policies, tax evasion, taxpayer audits are present.

Keywords: complex systems, economic inequality, income distribution, income redistribution, micro to macro models, tax audit, tax evasion, taxation, welfare.
\end{abstract}

\section{INTRODUCTION AND OUTLINE OF A GENERAL FRAMEWORK}

Intriguing examples of complex systems include monetary exchanges in a market society and the process leading to the formation of income and wealth distribution within a given population. Understanding this process at the "microscopic" level requires considering the interactions occurring between a large number of elementary units of the systems - individuals or households. Like for many complex systems, these units are not all equal, being characterized by different behaviour and attributes. The consequence is that even in the presence of well-defined rules governing the interactions, the observable "macroscopic" regularities cannot be easily inferred.

The recent financial and economic crisis, with its ongoing negative consequences, has highlighted the need for a new approach to the modelling of economic systems. Also the ensuing widespread rise in economic inequality has led to a vivid public debate, as testified by the success of some books which are dealing with this issue [1-5].

It provides an urgent motivation to gain further insight into the fundamental mechanisms that underlie the unfolding of macro-economic processes. The ultimate goal, of course, would be to figure out which concrete actions and policies one should adopt toward the attainment of a desired trend.

The observations above show the necessity of a perspective which recognizes the role of the heterogeneity of the units and the importance of their interconnections. Such a perspective differs from the traditional view of mainstream economics, centred on the assumption of a representative rational agent; it took shape essentially during the last two decades and has been developed mainly by means of agent-based computational methods. Related references in economics are for example [6-9]. A clear discussion of complexity in a general context can be found in [10], see also [11].

Within this general conceptual framework, the technical tool we employ is that of ordinary differential equation (ODE) systems. In the traditional literature, ODEs are typically associated with 
macroscopic phenomena, and accordingly, models are often described by only one or a few equations. In contrast, the model we are proposing, first developed in [12] and then variously extended and exploited e.g. in [13-16] to deal with different specific questions, is formulated by means of systems of several differential equations. These equations are nonlinear, of the kinetic discretized Boltzmann kind and involve in particular transition probabilities.

In the model, the individuals are divided into income classes and exchange money through binary and ternary interactions. The binary interactions provide a stylized representation of the payments producing income (the payment of an employer to a worker, of a patient to a physician, of a client to a lawyer and so on); the ternary interactions represent the effects of taxation and redistribution, processes which do not involve only the pair of directly interacting individuals (see [12] for the description of the mechanism). The differential equations which express the model are as many as the classes, distinguished by their average income. Let $n$ be their number.

The $j$-th equation describes the variation in time of the fraction $x_{j}$ of individuals belonging to the $j$-th class. In the basic version, it takes the form

$$
\frac{d x_{j}}{d t}=\sum_{h=1}^{n} \sum_{k=1}^{n}\left(C_{h k}^{j}+T_{[h k]}^{j}(x)\right) x_{h} x_{k}-x_{j} \sum_{k=1}^{n} x_{k},
$$

where

- the coefficients $C_{h k}^{j} \in[0,+\infty)$, satisfying $\sum_{j=1}^{n} C_{h k}^{j}=1$ for any fixed $h$ and $k$, express the probability that an individual of the $h$-th class will belong to the $j$-th class after a direct interaction with an individual of the $k$-th class;

- the functions $T_{[h k]}^{j}: \mathbf{R} n \rightarrow \mathbf{R}$ satisfy, for any fixed $h, k$ and $x \in \mathbf{R}^{n}, \sum_{j=1}^{n} T_{[h k]}^{j}(x)=0$. These functions account for the indirect interactions (taxation and redistribution) and express the variation in the $j$-th class due to an interaction between an individual of the $h$-th class with one of the $k$-th class.

Specific expressions for the $C_{h k}^{j}$ and $T_{[h k]}^{j}$ have been given in [12-16], also depending on the different questions therein tackled. In particular, these expressions contain parameters which can be variously tuned and represent encounter frequencies, saving propensities, average incomes, tax rates, and also tax evasion rates. In any case, the $C_{h k}^{j}$ and $T_{[h k]}^{j}$ are constructed in such a way that the vector $\left(x_{1}(t), \ldots, x_{\mathrm{n}}(t)\right.$ ) (suitably normalized) is a discretized probability density function at time $t$. We point out that in the cases studied in [12-16], as also here, the total wealth is assumed to remain constant in time. Growth or variation of the wealth, however, can be considered as well and are in fact the object of ongoing investigation.

Issues like redistribution through means-tested welfare policies, the impact of tax evasion on the income distribution and the effects of taxpayer audits have been treated in [12-16]. In all these cases simulations show that after a sufficiently long time the solution $\left(x_{1}(t), \ldots, x_{\mathrm{n}}(t)\right)$ of the equations reaches an equilibrium state which depends on the total income and on the interaction parameters, but not on the initial distribution. The histogram of its graphical representation gives the "asymptotic" income profile. We emphasize that this equilibrium solution does not prevent single individuals to change class; what is in equilibrium is the statistical distribution. Of course, in real world the situation is not exactly stationary, because parameters are subject to changes.

\section{SOME EXAMPLES OF APPLICATION OF THE OUTLINED FRAMEWORK}

Our aim in this section is to show how the general framework outlined above can be used to explore the scenarios evolving from different situations. To do that, we must go into some more detail. Referring also to [12-16] for extensive motivations and comments, we fix next the following terms. 
Let $S$ denote a unit amount of money, $S=0.1$ in numerical simulations. We define coefficients $p_{\mathrm{h}, \mathrm{k}}$ for $h, k=1, \ldots, n$, expressing the probability that in an encounter between an individual of the $h$-th and one of the $k$-th income class, the one who pays is the $h$-individual: we take $p_{h, k}=\min \left\{r_{h}, r_{k}\right\} / 4 r_{n}$, with the exception of the terms $p_{j, j}=r_{j} / 2 r_{n}$ for $j=2, \ldots, n-1, p_{h, 1}=r_{1} / 2 r_{\mathrm{n}}$ for $h=2, \ldots, n, p_{n, k}=$ $r_{k} / 2 r_{n}$ for $k=1, \ldots, n-1, p_{1, \mathrm{k}}=0$ for $k=1, \ldots, n$ and $p_{h, n}=0$ for $h=1, \ldots, n$. Then, we define the only possibly nonzero elements among the $C_{h k}^{j}$ as

$$
\begin{aligned}
C_{i+1, k}^{i} & =P_{i+1, k} \frac{S\left(1-T_{k}\right)}{r_{i+1}-r_{i}}, \\
C_{i+1, k}^{i} & =1-P_{k, i} \frac{S\left(1-T_{i}\right)}{r_{i+1}-r_{i}}-P_{k, i} \frac{S\left(1-T_{k}\right)}{r_{i}-r_{i-1}}, \\
C_{i-1, k}^{i} & =P_{k, i-1,} \frac{S\left(1-T_{i-1}\right)}{r_{i}-r_{i-1}},
\end{aligned}
$$

where $r_{j}$ represents the average income of the $j$-th class. In numerical simulations we take $r_{j}=25 j$ for $j=1, \ldots, n$.

We also take each $T_{[h k]}^{i}(x)$ as a sum $T_{[h k]}^{i}(x)=\mathrm{U}_{[h k]}^{i}(x)+V_{[h k]}^{i}(x)$ where

$$
U_{[h k]}^{i}(x)=\frac{p_{h, k} S_{T k}}{\sum_{j=1}^{n} w_{j} x_{j}}\left(\frac{w_{i-1} x_{i-1}}{r_{i}-r_{i-1}}-\frac{w_{i} x_{i}}{r_{i+1}-r_{i}}\right)
$$

and

$$
V_{[h k]}^{i}(x)=p_{h, k} S_{T k} \frac{\sum_{j=1}^{n-1} w_{j} x_{j}}{\sum_{j=1}^{n} w_{j} x_{j}}\left(\frac{\delta_{h, i+1}}{r_{h}-r_{i}}-\frac{\delta_{h, i}}{r_{h}-r_{i-1}}\right),
$$

with $\delta_{h, k}$ denoting the Kronecker delta, respectively, represent the variation density corresponding to the advancement (due to the benefit of redistribution) from a class to the subsequent one and to the retrocession (due to the payment of taxes) from a class to the preceding one. The coefficients $w_{\mathrm{j}}$ in (1) and (2) denote the weights of a differently distributed welfare. For simplicity, we let them be given by

$$
w_{j}=r_{n+1-j}+\frac{2}{n-1} \gamma\left(j-\frac{n+1}{2}\right)\left(r_{n}-r_{1}\right) \text {, }
$$

where $\gamma$ is a parameter taking values in $(0,1 / 2)$. The expression in (3) implies that the $w_{j}$ are decreasing in $j$ (decreasing linearly if $r_{j}$ is linear in $j$ ). By (3), one has $w_{1}-w_{n}=\left(r_{n}-r_{1}\right)(1-2 \gamma)$. Thus, if $\gamma=1 / 2, w_{1}=w_{n}$ holds true and $w_{j}$ has the same value for each $j=1, \ldots, n$; in contrast, $w_{1}-w_{n} \rightarrow r_{\mathrm{n}}$ $-r_{1}$ when $\gamma \rightarrow 0$.

We take the tax rates relative to the different classes as

$$
\tau_{j}=\tau_{\min }+\frac{j-1}{n-1}\left(\tau_{\max }-\tau_{\min }\right),
$$


for $j=1, \ldots, n$, where $\tau_{\max }$ and $\tau_{\min }$ respectively denote the maximum and the minimum tax rate. In numerical simulations, we take $\tau_{\text {min }}=23 \%$ and $\tau_{\text {max }}=43 \%$ (just because those are the minimum and maximum IRPEF tax rate in our country, Italy). To run simulations, we take here $n=15$.

Still, before moving to the discussion and comparison of some case studies, we point out that two indicators of interest in the economic context at hand are the Gini index and the tax revenue. The former, ranging between 0 and 1 , provides a measure of the deviation from a perfectly equal distribution. The latter coincides with the amount of money collected by the government through taxation and redistributed as welfare provision. We consider here the tax revenue in the unit time, calculated as

$$
T R=\sum_{h=1}^{n} \sum_{k=1}^{n} \sum_{j=1}^{n-1} p_{h k} \tau_{k} \frac{w_{j} \hat{x}_{j}}{\left(\sum_{i=1}^{n} w_{i} \hat{x}_{i}\right)} \hat{x}_{h} \hat{x}_{k},
$$

where $\hat{x}_{i}$ denotes the fraction of individuals in the $i$-th class at equilibrium.

We are now ready to investigate some variations of a specific fiscal policy case.

For the case, to which we refer as to the case $C_{0}$, characterized by the parameters above and by $\gamma=0.5$ we report in the first row of the Fig. 1 the histogram of the asymptotic stationary income distribution corresponding to a given initial condition (and, accordingly, to a given value of the total income). For the asymptotic distribution of $C_{0}$ we also calculate and report in Table 1 the Gini index $G$ and the tax revenue in the unit time $T R$. Then, we calculate and report in Table 1 the Gini index and the tax revenue of the asymptotic stationary income distribution corresponding to the same initial condition as for $C_{0}$, for the following partially varied situations:

1. a case $C_{1}$ differing from $C_{0}$ in the different values of $\tau_{\min }(20 \%$ instead of $23 \%)$ and $\tau_{\max }(65 \%$ instead of $43 \%$ );

2. a case $C_{2}$ encompassing provision of differentiated welfare for different income classes and in which, specifically, $\gamma=0.15$ (instead of $\gamma=0.5$ ). This means that the poorest class gets approximately four times what the richest class gets;

3. a case $C_{3}$ in which to some extent also tax evasion is present (specifically, half of the population is supposed to pay half of the due taxes);

4. a case $C_{4}$ in which both tax evasion and some audit procedure are postulated: specifically, one fifth of the population undergoes audit and a fine (to be paid in addition to the evaded amount) is applied to evaders, amounting to $3 / 4$ of the evaded amount.

Our focus is on a comparison between the asymptotic scenarios, as well as the values of $G$ and $T R$ associated to them, relative to the cases $C_{1}, C_{2}, C_{3}, C_{4}$ and that of the case $C_{0}$. We underline that it is not the parameters chosen that are especially significant. Rather, what is of interest is the possibility enhanced by the framework to explore different situations and establish comparisons between their features.

Table 1: The Gini index $G$ and the tax revenue $T R$ in five different cases.

\begin{tabular}{|c|c|c|}
\hline $\mathrm{C} 0$ & $G=0.379672$ & $T R=0.0175466$ \\
\hline $\mathrm{C} 1$ & $G=0.351037$ & $T R=0.022186$ \\
\hline $\mathrm{C} 2$ & $G=0.354214$ & $T R=0.0183758$ \\
\hline $\mathrm{C} 3$ & $G=0.403769$ & $T R=0.0011994$ \\
\hline $\mathrm{C} 4$ & $G=0.393106$ & $T R=0.00140869$ \\
\hline
\end{tabular}



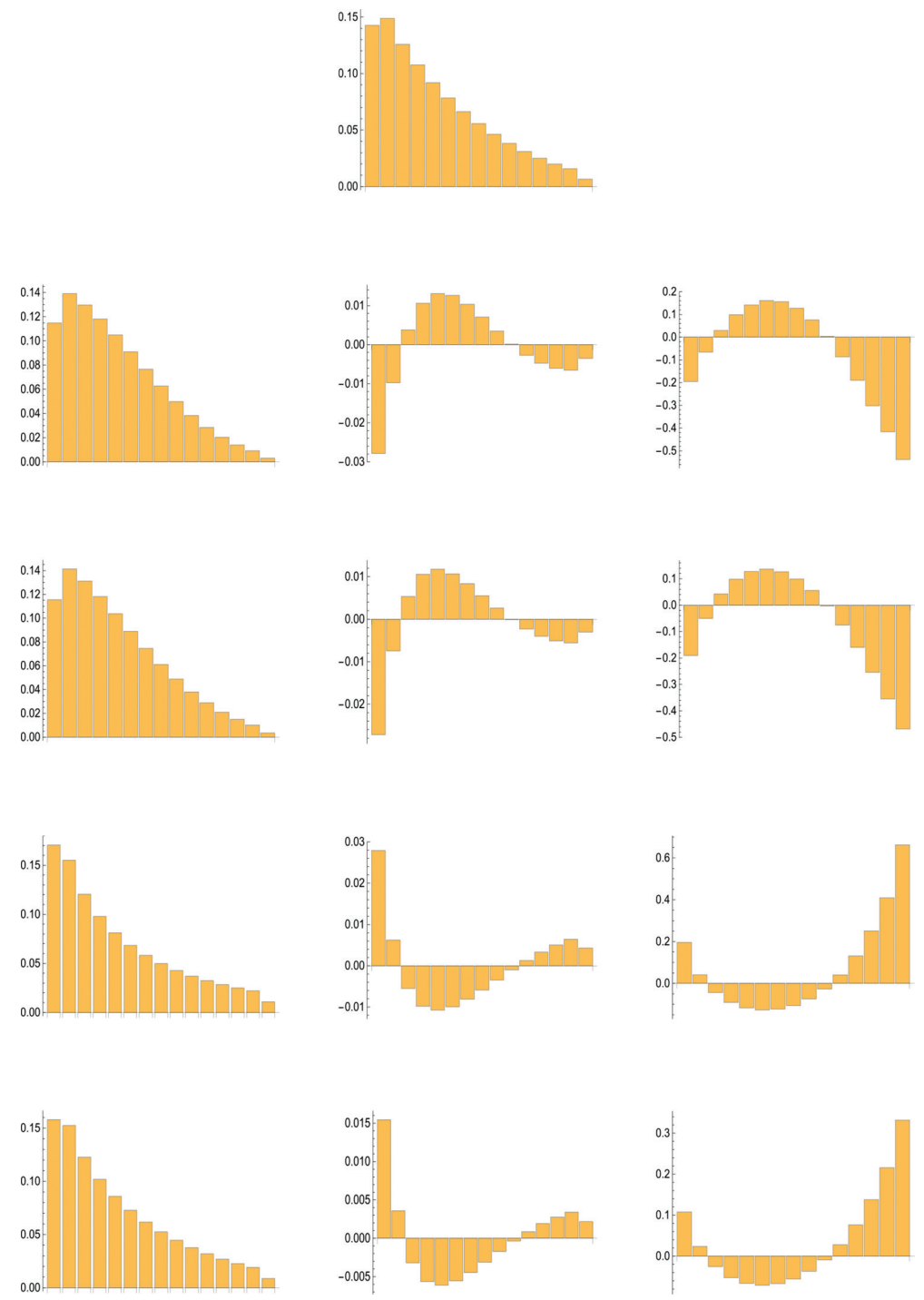

Figure 1: Asymptotic scenarios relative to five different cases.

An illustration of the different asymptotic scenarios is provided by Fig. 1. The rows from the second to the fifth in this figure respectively refer to the cases $C_{1}, C_{2}, C_{3}, C_{4}$. The histograms on the left represent the asymptotic stationary income distributions relative to the cases $C_{j}$ for $j=1,2,3,4$ and evolved from the same initial condition as the distribution in the first row (which is relative to $C_{0}$ ). The central histograms show the difference between the asymptotic distribution of $C_{j}$ (for $j=1,2,3$, 4) and that of $C_{0}$. The histograms on the right display the percentage of the difference between the asymptotic distribution of $\mathrm{C}_{j}$ and that of $C_{0}$. Notice that these histograms are differently scaled. What is evident at once is that, compared with the case $C_{0}$, both the cases $C_{1}$ and $C_{2}$ are characterized by a greater number of individuals in the middle classes and a smaller number of individuals in the 
poorest and the richest classes. In contrast, both for $C_{3}$ and $C_{4}$, the number of individuals in the poorest, as well as in the richest claases, is larger than in case $C_{0}$ and the contrary is true for the number of middle classes individuals. Accordingly, the Gini indices listed in Table 1 show that in the cases $C_{1}$ and $C_{2}$ economic inequality is smaller than in the case $C_{0}$, whereas in the cases $C_{3}$ and $C_{4}$ it is larger. The distinction between the features of the cases $C_{1}$ and $C_{2}$ on a side and $C_{3}$ and $C_{4}$ on another side holds true also for the values of the tax revenue: in the cases $C_{1}$ and $C_{2}$ these are larger than in the case $C_{0}$, in the cases $C_{3}$ and $C_{4}$ they are smaller. All the tax revenue values in Table 1 are pretty small, but one should keep in mind here that they just refer to a unit time.

In short, the results outlined look reasonably and qualitatively consistent with what one expects. It is not a surprise that welfare and a widening of the gap between the maximum and the minimum tax rates foster a decrease of inequality. Analogously, it is known that tax evasion is harmful to the collectivity. What our approach provides in addition is quantitative information. It may then be used, of course in combination with real data, to get insights as how to fix parameters (tax rates, fraction of audited individuals, fines, ...) to obtain some desired output.

\section{CONCLUSIONS AND FURTHER RESEARCH}

In this paper, we presented and compared the outputs of some versions of a model which is able to explain the emergence of aggregate-level regularities of economic processes (such as the income distribution of a population) out of a whole of interactions occurring at the individual level. The interactions we took into consideration include money exchanges, payment of taxes, redistribution of benefits, effects of means-tested welfare policies, tax evasion, taxpayer audit and payment of fines. We explored in particular the difference of long run evolutions of the income distribution taking place in the presence of different conditions. Our findings are consistent with the expectation based on empirical observations. This suggests that the model could give some insights towards the adoption of suitable policies. In fact we believe that its main value lies in its adaptability to different problems and data and in its forecasting ability.

There are now several directions, along with further investigation can be carried out. An important aspect concerns for example the introduction into the model of a network structure. We devote the following lines to a preliminary discussion of this issue.

In our discretized kinetic models, individuals are collected in classes according to their income. Likewise, in discretized kinetic models of particle systems in physics, particles are generally collected in discrete energy classes. The discretization allows to convert the integro-differential equations of the Boltzmann kind typical of continuum kinetic theory into a system of coupled ordinary differential equations which are easier to solve numerically and allow a greater degree of flexibility in the definition of the interactions. The number of classes (typically 10-30 in our numerical solutions) can be increased for better accuracy, but in fact it is not necessary to attain large values of $n$, because in an economic system the continuum limit is not generally needed; on the contrary, most real data on income distributions and taxation are structured according to income classes. On the other hand, it may be desirable to introduce different kinds of heterogeneity. When the model is applied to the description of tax evasion, for instance, each income class can be further subdivided into classes of individuals with different evasion behaviour (total evaders, partial evaders, honest etc.; in the previous section: partial evaders and honest). Another possibility is to consider a subdivision of each income class into "link classes", or classes of individuals with different numbers of economic connections to other individuals. This amounts to the introduction of a network structure, a procedure which has proven to be very fruitful in many dynamical models based on differential equations. It is clear that economic systems possess a network structure, with individuals having preferential interactions along certain links which can be fixed or vary in time. 
A first step we propose here is to consider a fixed network structure and employ a statistical representation of the network as introduced by Boguna et al. [17] for the study of epidemics. Within this representation the link density function denoted by $\mathrm{P}(\alpha)$ would represent the fraction of all individuals who have $a$ links of economic nature to other individuals and the correlation function denoted by $P(\beta \mid \alpha)$ would represent the conditional probability for an individual with $\alpha$ links to be connected to an individual with $\beta$ links. In the case of uncorrelated networks the function $\mathrm{P}(\beta \mid \alpha)$ is just expressed in terms of $P(\beta)$, since $P(\beta / \alpha)=\beta P(\beta) / \sum_{\gamma=1}^{N} \gamma P(\gamma)$, with $\mathrm{N}$ denoting the maximum number of links. Otherwise, it is an independent quantity, although the so-called network closure condition $\alpha P(\beta \mid \alpha) P(\alpha)=\beta P(\alpha \mid \beta) P(\beta), \forall \alpha, \beta=1, \ldots, N$ [18] always must hold true. Further, more technical conditions upon the kinetic equations (compare [15] for details) come from the fact that in models for economic exchanges money is conserved (while in a contagion process, unfortunately, the disease can multiply for free). Broadly speaking, the correlations can be of the assortative or disassortative kind. In the assortative case, individuals with many links are more likely to be connected to other individuals with many links; in the disassortative case, the opposite happens. In the presence of a network structure, the class population variables $x_{i}(t)$ which give the fraction of individuals belonging to the income class $i$ at time $t$ will become $x_{i}^{a}(t)$, so that $x_{i}^{a}(t)$ represents the fraction of individuals who have $\alpha$ links (fixed) and belong at time $t$ to the income class $i$. In the asymptotic limit $t \rightarrow \infty$ one can expect that the income distributions corresponding to different numbers of links will be different. It makes sense, for instance, to compare the asymptotic income distribution $\left\{\hat{x}_{1}^{1}, \hat{x}_{2}^{1}, \ldots, \hat{x}_{n}^{1}\right\}$ of individuals with only 1 link to the distribution $\left\{\hat{x}_{1}^{N}, \hat{x}_{2}^{N}, \ldots, \hat{x}_{n}^{N}\right\}$ of individuals with $N$ links. One might expect that "more links will make you richer", on the average, and therefore in the latter income distribution the richer classes will be more populated and the average income higher. This actually happens in some cases, but not always, as the outcome of the model generally depends on the structure of the network and in particular on the exponent $q$ in the important case of scale-free networks with link density $P(\alpha) \propto \alpha^{-\mathrm{q}}$. Carrying out this program in a practical situation is not straightforward and requires a considerable preliminary work. Indeed, one has to find reasonable values of the elements of the correlation matrix, which have to satisfy the above-mentioned technical conditions too. This will be for us the object of future investigation.

\section{REFERENCES}

[1] Wilkinson, R. \& Pickett, K., The Spirit Level. Why Equality is Better for Everyone, Penguin Books: London, 2010.

[2] Milanovic, B., The Haves and the Have-Nots: A Brief and Idiosyncratic History of Global Inequality, Basic Books: New York, 2012.

[3] Stiglitz, J.E., The Price of Inequality: How Today's Divided Society Endangers Our Future, W.W. Norton \& Company: New York, 2012.

[4] Piketty, T., Capital in the Twenty-First Century, Harvard University Press: Cambridge, 2014.

[5] Atkinson, A.B., Inequality: What Can Be Done? Harvard University Press: Cambridge, 2015.

[6] Arthur, B., Durlauf, S. \& Lane, D.A., Process and the emergence in the economy. In The Economy as an Evolving Complex System II, eds. B. Arthur, S. Durlauf \& D.A., Lane, Addison-Wesley: Reading MA, 1997.

[7] Kirman, A., Complex Economics: Individual and Collective Rationality, Routledge: London, 2010.

[8] Gallegati, M. \& Kirman, A., Reconstructing economics: agent based models and complexity. Complexity Economics, 1, pp. 5-31, 2012.

http://dx.doi.org/10.7564/12-COEC2 
[9] Tesfatsion, L. \& Judd, K.L. (eds), Handbook of Computational Economics, Vol. 2: Agentbased Computational Economics, North-Holland, Amsterdam, 2006.

[10] Rzevski, G., Complexity as the defining feature of the 21 st century. International Journal of Design \& Nature and Ecodynamics, 10, pp. 191-198, 2015. http://dx.doi.org/10.2495/DNE-V10-N3-191-198

[11] Rzevski, G. \& Brebbia, C.A. (eds), Complex Systems: Fundamentals \& Applications, WIT Press: Accurst, 2016.

[12] Bertotti, M.L., Modelling taxation and redistribution: a discrete active particle kinetic approach. Applied Mathematics Computation, 217, pp. 752-762, 2010. http://dx.doi.org/10.1016/j.amc.2010.06.013

[13] Bertotti, M.L. \& Modanese, G., Micro to macro models for income distribution in the absence and in the presence of tax evasion. Applied Mathematics Computation, 244, pp. 836-846, 2014. http://dx.doi.org/10.1016/j.amc.2014.07.055

[14] Bertotti, M.L. \& Modanese, G., Microscopic models for welfare measures addressing a reduction of economic inequality. Complexity, in print; article first published online March 2015. http://dx.doi.org/10.1002/cplx.21669

[15] Bertotti, M.L. \& Modanese, G., Discretized kinetic theory on scale-free networks. EPJ ST, in print, available on arXiv:1503.03744.

[16] Bertotti. M.L. \& Modanese, G., Microscopic models for the study of taxpayer audit effects. International Journal Modern Physics C, in print, available on arXiv:1602.08467

[17] Boguna, M., Pastor-Satorras, R. \& Vespignani, A., Absence of epidemic threshold in scale-free networks with degree correlations. Physics Review Letters, 90, 2003.

[18] Serrano, M.A., Boguna, M., Pastor-Satorras, R. \& Vespignani, A., Correlations in complex networks. In Large Scale Structure and Dynamics of Complex Networks: from Information Technology to Finance and Natural Sciences, eds. G. Caldarelli \& A. Vespignani, World Scientific: New Yersey, 2007.

http://dx.doi.org/10.1142/9789812771681_0004 